REVIEW

\title{
THE ROLE OF RNA METABOLISM IN NEUROLOGICAL DISEASES
}

\author{
Alaqeel AM ${ }^{1,2, *}$, Abou Al-Shaar $\mathrm{H}^{3}$, Shariff RK ${ }^{3}$, Albakr A ${ }^{2}$
}

*Corresponding Author: Ahmed M. Alaqeel, M.D., Department of Neurosurgery, University of Calgary, 1403 29th Street NW, Calgary, Alberta T2N 2T9, Canada. Tel: +403-970-9117. Fax: +403-270-7878. E-mail: ahmedalaqeel@hotmail.com

\begin{abstract}
Neurodegenerative disorders are commonly encountered in medical practices. Such diseases can lead to major morbidity and mortality among the affected individuals. The molecular pathogenesis of these disorders is not yet clear. Recent literature has revealed that mutations in RNA-binding proteins are a key cause of several human neuronal-based diseases. This review discusses the role of RNA metabolism in neurological diseases with specific emphasis on roles of RNA translation and microRNAs in neurodegeneration, RNA-mediated toxicity, repeat expansion diseases and RNA metabolism, molecular pathogenesis of amyotrophic lateral sclerosis and frontotemporal dementia, and neurobiology of survival motor neuron (SMN) and spinal muscular atrophy.
\end{abstract}

Keywords: Amyotrophic lateral sclerosis; Frontotemporal dementia; RNA; Survival motor neuron (SMN); Spinal muscular atrophy.

\section{INTRODUCTION}

Neurodegenerative diseases are one of the leading health challenges in modern medical history because of their intriguing nature and the lack of knowl-

\footnotetext{
${ }^{1}$ Department of Neurosurgery, University of Calgary, Calgary, Alberta, Canada

${ }^{2}$ Division of Neurosurgery, Department of Surgery, King Saud University, Riyadh, Saudi Arabia

${ }^{3}$ College of Medicine, Alfaisal University, Riyadh, Saudi Arabia
}

edge in the understanding of the underlying causes. Recent research has helped scientists acquire a greater insight into the cellular, molecular and genetic predispositions of these conditions. The most difficult aspect in treating these diseases is the time required to make a proper diagnosis, which is usually when the disease has already advanced to a stage of irreparable damage. The studies carried out in the last 15 years have helped scientists gain a better insight into the molecular and genetic levels of predisposing factors and disease mechanisms. Once the actual cause and the pattern of disease progression are discovered, the treatment options will become more available and effective.

RNA metabolism is the basic process by which RNA is generated, transported, regulated, stored and translated. Recent studies have shown that mutations in RNA-binding proteins are a key cause of several human neuronal based diseases.

The major contributing factors to these neurodegenerative diseases include pathogenic mutations, amyloid precursor proteins, superoxide dismutase, and DNA and RNA binding proteins. Additionally, the most prominent factor associated with these diseases is age. Studies have linked several genetic risk factors with several neurological diseases, which predispose individuals to develop these conditions $[1,2]$. Table 1 depicts the role of RNA in various neurological diseases. The literature states that there are some common molecular events that happen during the development and progression of these neurodegenerative disorders, whether similar or different. 
Table 1. RNA role in several neurological diseases.

\begin{tabular}{|l|l|}
\hline Disease & Cause \\
\hline Alzheimer's disease & TDP-43, mir-137/181b, BACE1AS \\
\hline Down syndrome & TDP-43 \\
\hline Frontotemporal lobar dementia & TDP-43 \\
\hline Amyotrophic lateral sclerosis & TDP-43, mir-206 \\
\hline Familial British dementia & TDP-43 \\
\hline Parkinson's disease & TDP-43, BC200, SOX20T, mir-34b/c, mir-153 \\
\hline Huntington's disease & HAR1, DGCR5, TDP-43, FUS/TLS, TUG1, NEAT 1, toxic RNA, mir-132, mir-7, mir-34b \\
\hline Spinocerebellar ataxia 1,2,3,4 & FUS/TLS \\
\hline Spinocerebellar ataxia 10, 12 & Toxic RNA, RAN (repeat associated non ATG translation), expansion in non coding region \\
\hline Dentatorubral-pallidoluysian atrophy & FUS/TLS \\
\hline Myopathies & TDP-43 \\
\hline Fragile X tremor ataxia syndrome & Antisense transcription, expansion in non coding region \\
\hline X-linked mental retardation & UPF3B mutation \\
\hline Autism & 7q22-q33 (non coding RNA) \\
\hline Spinal muscular atrophy & Mutation in SMN2 \\
\hline Spinocerebellar ataxia & Non coding RNA \\
\hline Myotonic dystrophy type 1 (DM1) & RAN (repeat associated non ATG translation), expansion in non coding region \\
\hline Myotonic dystrophy type 2 (DM2) & Expansion in non coding region \\
\hline
\end{tabular}

TDP-43: trans-activation response (TAR) element DNA-binding protein 43; miR: microRNA; HAR1: human accelerated region 1; DGCR5: DiGeorge syndrome critical region gene 5; FUS/TLS: fused in sarcoma/translocated in sarcoma; TUG1: taurine up-regulated 1; NEAT1: nuclear enriched abundant transcript 1; RAN: repeat-associated non-ATG; UPF3B: UPF3 regulator of nonsense transcripts homolog B; SMN2: survival of motor neuron 2.

One of the common molecular events is oxidative stress, which affects protein folding, aggregation and degradation of some protein species, lipid peroxidation, calcium homeostasis and DNA repair. Molecular events also include protein oligomerization and aggregation, axonal transport deficits, mitochondrial dysfunction, excitotoxicity, calcium dysregulation, neuron-glial interactions, neuroinflammation, DNA damage and aberrant RNA processing.

RNA Translation in Neurodegeneration. Some researchers have proposed that the synaptic efficacy of neuronal dendrites and dendritic spines may depend on local (synaptodendritic) protein synthesis due to the presence of mRNA and polyribosomes [3]. In situ hybridization studies have further strengthened the concept of local translation by demonstrating the selective localization of some mRNA transcripts to dendrites. These studies now suggest that certain RNA forms are packaged into discrete ribonucleoproteins (RNPs) that are then transported to the dendrites where they are selectively expressed [4].
Synapses and synaptic networks are affected by local translational control. This is demonstrated in different studies wherein synapse-stimulating agents have been found to elicit protein synthesis [5], while protein synthesis inhibitors disrupt synaptic plasticity including long-term potentiate (LTP), long-term depression (LTD), and long-term facilitation [6]. Long-lasting late-phase LTP (L-LTP) is a form of LTP that requires both gene transcription and RNA translation [7].

The three major factors that control translation in neurons, namely miRNAs, fragile X mental retardation (FMRP), and cytoplasmic polyadenylation [poly(A)] element-binding (CPEB) proteins are now being investigated in detail to understand their mechanisms and interactions [8]. Cytoplasmic poly(A) element-binding is a sequence-specific RNA-binding protein that represses translation until stimulated. Once stimulated by signaling events, it leads to translation activation by elongation of poly(A) tails of mRNA [9]. Another associated factor is cytoplasmic 
poly(A) complex, which in turn affects poly(A)-induced translation of mRNAs in dendrites in response to synaptic stimulation [10]. The importance of these cytoplasmic poly(A) complexes in synaptic function can be observed by the changes in synaptic plasticity, which occur in response to depletion of poly(A) complex factors [9]. The CPEB displays a coherent posttranscriptional molecular mechanism that underlies essential brain functions.

RNA-Mediated Toxicity. Unstable trinucleotide repeat expansions in the associated genes have been found to be the cause of a large number of neurodegenerative diseases [11]. These disorders can be classified into different groups based on the pathogenic mechanisms such as RNA toxicity, loss of protein function and dominant protein-based toxicity.

RNA toxicity has been found to be responsible for two neurodegenerative diseases named myotonic dystrophy type 1 (DM1) and myotonic dystrophy type 2 (DM2). The CUG and CCUG RNA expansions in the non coding regions of the dystrophia myotonica-protein kinase (DMPK) gene and the zinc finger 9 gene (ZNF9), result in DM1 and DM2, respectively [12].

Polyglutamine (polyQ) diseases such as Huntington's disease (HD) and the spinocerebellar ataxias (SCAs) (such as SCA1, 2, 3, 6, 7 and 17) are inherited disorders caused by expanded CAG repeats within the coding region of the associated genes [13]. A prominent pathological trait of these diseases is the ubiquitinated nuclear inclusions (NIs) containing chaperones and subunits of the proteasome [13]. Research has predominantly focused on protein-based mechanisms of toxicity caused by the expanded polyQ domain. Recent studies are now suggesting that RNA toxicity is the principal reason for degeneration [14]. In one study, it was observed that the toxicity of a pathogenic expanded polyQ Ataxin-3 protein was significantly contained by interrupting the typically pure CAG-repeat sequence encoding the polyQ domain with a CAA-interrupted repeat that also encodes for glutamine. It has been proposed that the toxicity is caused by the hairpin structure formed by the CAG-repeat of RNA [15]. Interrupting the CAG-repeat RNA by CAA codons has been indicated to disrupt the hairpin structure [16]. Some old studies have shown that pure or interrupted CAG-repeat expansions in ATAXIN-2 (ATXN2) are linked with distinct effects [17]. Expanded pure CAG repeat in
ATXN2 typifies SCA2. CAA interruptions in ATXN2 have been found in CAG-repeat expansions that are associated with amyotrophic lateral sclerosis (ALS) and Parkinsonism. These findings clearly indicate the significance of the CAG-repeat RNA in toxicity and its effects. Studies also revealed that a non coding RNA bearing only an abnormally long CAG repeat, but not coding for a protein, can result in progressive neuronal dysfunction including shortened life span and locomotor defects [11].

A set of studies with drosophila have shown that muscleblind (mbl), an RNA-binding protein (implicated as a modifier in DM1), can enhance the toxicity of both Ataxin-3 protein and non coding CAG-repeat RNA [18]. These observations clearly indicate that the CAG-repeat RNA, in the absence of coding for a protein, can become a source of toxicity in polyQ diseases.

Roles of MicroRNAs in Neurodegeneration. It is now an established fact that microRNAs (miRNAs) have a significant role in neurodegeneration $[19,20]$. Micro-RNAs are the small endogenous RNAs that act as post-transcriptional regulators of gene expression. MicroRNAs primarily serve as guide molecules for miRNA-containing ribonucleoprotein (miRNP) complexes to reach their target mRNA. The binding of the miRNP to the target in turn affects the expression of the target mRNA [21]. The target levels of expression are reduced either by the inhibition of the translation of the target mRNA or by the destabilization caused by mRNA deadenylation [22]. There have been observations of up-regulation of targets by miRNA [23].

Studies indicate that miRNAs have a huge regulatory potential as they target hundreds of proteincoding genes $[24,25]$. Many miRNAs are expressed in the central nervous system in a temporally- and/ or spatially-regulated manner during development [26]. Research has estimated that $>1000$ miRNAs are expressed in the human brain [26].

Recent years have seen a rise in research on miRNAs and their role in normal development, differentiation, functions, as well as in pathology. MicroRNA target sites are now being recognized for single nucleotide polymorphisms (SNPs) associated with various neural diseases [27]. MicroRNAs provide the sequence information that guides the miRNP to target mRNAs. Base-pairing interactions involving six to eight residues at the 5' end of the miRNA, 
called the "seed", are thought to provide most of the target recognition specificity with additional basepairings contributing to the magnitude of regulation [25]. Target sites are mainly observed in the non coding portion of mRNAs [in 3' untranslated regions (3'UTRs)]. Change in one residue in a 3'UTR result in a new seed match that leads to reduced expression of that allelic form of the mRNA. Similarly, a loss of a miRNA site could be caused by a 3'UTR SNP, which in turn will result in an elevated expression of that allele. A study identified an SNP that creates a site for human miR-659 in the 3'UTR of the progranulin gene, as a risk factor in a specific form of dementia [28]. Another study reported an SNP that destroys a site for miR-433 in the 3'UTR of FGF20 and has been linked to an increased risk of Alzheimer's disease (AD) caused by overexpression of a-synuclein [29].

Research studies are now progressively implicating miRNAs in biological processes linked to neurodegenerative diseases. An occurrence of the polyQ repeats in many proteins has been found to be directly linked to neurode-generation [30]. Bilen et al. [31] reported that depletion of dicer in HeLa cells significantly enhanced pathogenic Ataxin-3-induced toxicity. The adverse effects could be partially reduced by complementing the cells with the purified small RNA fraction containing total HeLa cell miRNAs [31]. The polyQ disorder SCA1 results in the death of cerebellar Purkinje cells [30]. In a research study, depletion of dicer from mouse Purkinje neurons did not impair cell survival in young mice, but when they aged beyond 13 weeks, Purkinje neurons began to die with degeneration and apoptosis. These mice displayed mild ataxia that aggravated with age [32].

MicroRNAs have also been associated with loss of dopaminergic neurons in Parkinson's disease (PD). MiR-133b was found to be expressed at reduced levels in brains and in animal models with PD [33]. Overexpression of miR-133 inhibited the differentiation of embryonic stem cells into dopaminergic neurons, while its depletion, using antisense oligonucleotides, increased the expression of dopaminergic neuronal markers. The transcription factor pitx 3 works as a target for miR-133b, confirming that pitx 3 thus regulates miR-133b expression, providing a feedback loop, which is essential for normal control of gene expression in this important cell type. Whether misregulation of miR-133b is linked to clinical disease remains to be determined.
Patients with dentatorubral-pallidoluysian atrophy (DRPLA) have expanded polyQ repeats of human Atrophin-1, which are the reason for neuronal apoptosis. In drosophila studies, it was found that miR-8 sets the level of Atrophin expression by binding to its 3'UTR. Experimental drosophila lacking miR-8 performed poorly in an assay for motor coordination and their performance gradually declined with age. There were abnormal cell deaths observed in the brains of these flies. These defects were amended by restricting the degree to which Atrophin is overexpressed, which in turn indicates that elevated Atrophin levels are responsible for the neurodegenerative defects [34]. The miR-8 in drosophila is related to human miR-200b and miR-429, which are known to target the Atrophin orthologue arginine-glutamic acid dipeptide repeats encoded (RERE) [34]. This RERE has been demonstrated to bind to Atrophin-1 resulting in its overexpression and subsequent apoptosis in neuronal cell cultures [35].

\section{Repeat Expansion Diseases and RNA Metabo-}

lism. The expansion of the polymorphic repeat sequences has been found to be the causative agent for over 20 human diseases. It beholds several types of SCA, HD and myotonic dystrophy [36]. The patients inherit a repeat number that exceeds the pathogenic threshold. The age-of-onset and the severity of diseases are associated with the repeat length expansion that happens within successive generations [36]. The expansion is caused by the loss of repeat-containing gene function, and recessive inheritance, and by the dominant repeat-mediated mechanisms [37]. Many observations signify mutational causes in multiple related neurodegenerative diseases. These include similar genetic and clinical features [30,36].

In many of the neurodegenerative diseases, the expanded CAG trinucleotide repeats are identified within a coding region. This results in an expanded polyQ tract in the final protein [36]. Polyglutamine can cause abnormalities due to the gain-of-function interactions inherent to the expanded protein tract and alterations to normal protein function [38]. However, in some disorders, repeats do not encode polyQ, share similar repeat sequences, pathogenic thresholds, or clinical features with the 'polyQ' diseases. In these diseases, the gain-of-function properties of the expanded repeat RNA transcript is the main source for the pathology, moreover, it consists of diseases such as Huntington's disease-like-2 (HDL-2), DM1 and DM2, 
SCA $8,10,12$, and fragile $\mathrm{X}$ tremor/ataxia syndrome (FXTAS) [11,39]. Some animal studies demonstrate how the repeat RNA is able to cause pathology in the animals as well as cellular model systems, through multiple and possibly distinct pathways [14,17].

Pathology caused by expanded repeat RNA was first reported in DM1. These pathologies were caused by a non coding CUG repeat within the 3'UTR of the $D M P K$ gene. In DM2, it was found to be caused by a non coding CCUG repeat within an intron of ZNF9 (CNBP) [40]. These expanded repeat RNA transcripts form a 'hairpin' secondary structure that binds and sequesters specific RNA-binding proteins [18]. The most extensive characteristic of these is mbl-like 1 (MBNL1), which is involved in the regulation of alternative splicing [41]. Sequestration of MBNL-1 results in the missplicing of certain transcripts and subsequently resulting in disease [12]. The pathology is associated with the formation of nuclear RNA foci that colocalize with MBNL-1 and are the sites at which sequestration occurs $[18,42]$. Some other proteins have also been identified that are bound or misregulated by repeat RNA, including Pur alpha [43], CUG-binding protein (CUG-BP) [44], and some heterogeneous nuclear ribonucleoproteins (hn RNPs) [45].

The colocalization of MBNL-1 with RNA foci and the dysregulation of alternative splicing have been observed in human tissues and animal models of the untranslated repeat diseases such as SCA10 [45], DM1, DM2 [12], SCA8 [46], FXTAS [47], and HDL-2 [48]. The sequestration of MBNL-1 and dysregulation of splicing may be a contributing factor to many disorders. Moreover, RNA foci that colocalizes with MBNL-1 is observed in human HD cells [48] It further emphasizes the supporting role for this pathway in the polyQ diseases.

Studies have identified the RNA repeats distinct from RNA-binding protein sequestration that can result in different disorders. Some studies have shown the pathogenic role of bi-directional transcription; it produces complementary repeat transcripts that hybridize to form double-stranded RNA [17]. Modification in Dicer-2 levels has been found to affect the pathology and is associated with the formation of 21 nucleotide repeat RNAs, highlighting a role for small RNA processing pathways. Bi-directional transcription is involved in a number of diseases and this pathway may be a common contributor to a dominant pathology [49].
Another alternative mechanism that may be responsible for some pathologies is the non ATG initiated translation in all three reading frames [50]. This process combined with bi-directional repeat transcription may be a contributing factor in many pathologies through the production of multiple homopolymeric proteins [50]. As the expanded repeat disease involves late onset of neurodegeneration and loss of affected tissue, access to samples and the detection of early changes in pathology becomes difficult. Therefore, human studies are limited and animal models became an essential in investigating pathology. Drosophila is a good model for expanded repeat disease and shares a number of key pathways that are similar to that of humans $[14,17,38,51]$. The studies with these models have shown that repeat RNA transcripts may contribute to dominantly inherited human pathology through multiple pathways [17].

Frontotemporal dementia (FTD) and ALS have similar genetic, clinical, and pathologic features [52]. The majority of patients with FTD develop features of motor neuron dysfunction [52], and nearly half of those with ALS are found to have evidence of frontal lobe impairment [52]. Amyotrophic lateral sclerosis is a fatal degenerative illness primarily affecting motor neurons, while FTD is a progressive dementing illness affecting neurons of the frontal, insular, and anterior temporal cortex [36]. Some individuals with FTD may incline to develop ALS [53]. The common factor in the pathogenesis of the two diseases is found to be trans-activation response (TAR) element DNA-binding protein 43 (TDP-43) [54]. Both ALS and FTD are characterized by abnormal accumulations of TDP-43 [37]. The most common pathological subtype of FTD is referred to as frontotemporal lobar degeneration with TDP-43 (FTLD-TDP) [55].

A number of families have been reported with an autosomal dominant pattern of disease in which affected members may develop either FTD, ALS, or both (FTD-ALS). Many of these families show a genetic linkage to a region on chromosome 9p21 [56]. This chromosomal region is identified with both ALS and FTD in many research studies. This illustrates the genetic defect at chromosome $9 \mathrm{p}$ may be responsible for both FTD and ALS [57,58].

The expansion of GGGGCC repeats is the most common genetic cause of ALS and FTD with a prevalence of approximately $5.0-7.0 \%$ in European and North American cohorts [59]. It accounts for approxi- 
mately $40.0 \%$ of familial ALS and $20.0 \%$ of familial FTD. The age-related progression of ALS and FTD along with the noncoding nature of the GGGGCC expansion is very much similar to other noncoding nucleotide repeat disorders, such as myotonic dystrophy, FXTAS, and SCAs 8, 10 and 12.

\section{CONCLUSIONS}

RNA has an extremely important role in various functions of the body. It has made a significant contribution toward the normal function of the neural system. Disrupted functions of RNAs and RNPs are a source of numerous maladies, including neurodegenerative diseases. Various ways have been proposed by which RNA and related proteins can lead to these disorders. The intricate process of alternative splicing provides incredible advantages for generating transcriptome and proteome diversity. It is also responsible for the risk of malfunction. It is essential that eliminating or redirecting the splicing of pre-mRNA results in an effective restoration of the activity and reduction of RNA toxicity. This clearly shows that these RNAs have a greater role in these degenerative diseases than what was thought before. Further studies will reveal more complex details, which will result in a better understanding of the treatment modalities that can be adopted. There is a need for a larger investment of resources in this area to understand the mysterious domain of these neurodegenerative diseases. It has truly left the medical world striving to unravel the deep secrets behind the molecular and genetic levels in them.

Declaration of Interest. This study was funded by the College of Medicine Research Center, Deanship of Scientific Research, King Saud University, Riyadh, Saudi Arabia. The authors have no personal financial or institutional interest in any of the drugs, materials, or devices described in this article. The authors have read and approved the final version of the submitted article. The authors report no conflicts of interest. The authors alone are responsible for the content and writing of this article.

\section{REFERENCES}

1. Crosiers D, Theuns J, Cras P, Van Broeckhoven C. Parkinson disease: Insights in clinical, genetic and pathological features of monogenic disease subtypes. J Chem Neuroanat. 2011; 42(2): 131141.

2. Ross CA, Tabrizi SJ. Huntington's disease: From molecular pathogenesis to clinical treatment. Lancet Neurol. 2011; 10(1): 83-98.

3. Steward O, Schuman EM. Protein synthesis at synaptic sites on dendrites. Annu Rev Neurosci. 2001; 24: 299-325.

4. Martin KC, Ephrussi A. mRNA localization: Gene expression in the spatial dimension. Cell. 2009; 136(4): 719-730.

5. Jiang C, Schuman EM. Regulation and function of local protein synthesis in neuronal dendrites. Trends Biochem Sci. 2002; 27(10): 506-513.

6. Martin KC, Casadio A, Zhu H, Yaping E, Rose JC, Chen M, et al. Synapse-specific, long-term facilitation of aplysia sensory to motor synapses: A function for local protein synthesis in memory storage. Cell. 1997; 91(7): 927-938.

7. Klann E, Dever TE. Biochemical mechanisms for translational regulation in synaptic plasticity. Nat Rev Neurosci. 2004; 5(12): 931-942.

8. Schratt G. MicroRNAs at the synapse. Nat Rev Neurosci. 2009; 10(12): 842-849.

9. Richter JD, Klann E. Making synaptic plasticity and memory last: Mechanisms of translational regulation. Genes Dev. 2009; 23(1): 1-11.

10. Wu L, Wells D, Tay J, Mendis D, Abbott MA, Barnitt A, et al. CPEB-mediated cytoplasmic polyadenylation and the regulation of experience-dependent translation of alpha-CaMKII mRNA at synapses. Neuron. 1998; 21(5): 11291139.

11. Li LB, Bonini NM. Roles of trinucleotide-repeat RNA in neurological disease and degeneration. Trends Neurosci. 2010; 33(6): 292-298.

12. Ranum LP, Cooper TA. RNA-mediated neuromuscular disorders. Annu Rev Neurosci. 2006; 29: 259-277.

13. Gatchel JR, Zoghbi HY. Diseases of unstable repeat expansion: Mechanisms and common principles. Nat Rev Genet. 2005; 6(10): 743-755.

14. Li LB, Yu Z, Teng X, Bonini NM. RNA toxicity is a component of ataxin-3 degeneration in Drosophila. Nature. 2008; 453(7198): 1107-1111. 
15. Sobczak K, de Mezer M, Michlewski G, Krol J, Krzyzosiak WJ. RNA structure of trinucleotide repeats associated with human neurological diseases. Nucleic Acids Res. 2003; 31(19): 54695482.

16. Sobczak K, Krzyzosiak WJ. CAG repeats containing CAA interruptions form branched hairpin structures in spinocerebellar ataxia type 2 transcripts. J Biol Chem. 2005; 280(5): 3898-3910.

17. Yu Z, Teng X, Bonini NM. Triplet repeat-derived siRNAs enhance RNA-mediated toxicity in a Drosophila model for myotonic dystrophy. PLoS Genet. 2011; 7(3): e1001340. doi: 10.1371/journalpgen. 1001340.

18. Miller JW, Urbinati CR, Teng-Umnuay P, Stenberg MG, Byrne BJ, Thornton CA, et al. Recruitment of human muscleblind proteins to (CUG) (n) expansions associated with myotonic dystrophy. EMBO J. 2000; 19(17): 4439-4448.

19. Bushati N, Cohen SM. MicroRNAs in neurodegeneration. Curr Opin Neurobiol. 2008; 18(3): 292-296.

20. Hébert SS, De Strooper B. Alterations of the microRNA network cause neurodegenerative disease. Trends Neurosci. 2009; 32(4): 199-206.

21. Bartel DP. MicroRNAs: Target recognition and regulatory functions. Cell. 2009; 136(2): 215233.

22. Filipowicz W, Bhattacharyya SN, Sonenberg N. Mechanisms of post-transcriptional regulation by micro-RNAs: Are the answers in sight? Nat Rev Genet. 2008; 9(2): 102-114.

23. Vasudevan S, Tong Y, Steitz JA. Switching from repression to activation: MicroRNAs can upregulate translation. Science. 2007; 318(5858): 1931-1934.

24. Krek A, Grün D, Poy MN, WolfR, Rosenberg L, Epstein EJ, et al. Combinatorial microRNA target predictions. Nat Genet. 2005; 37(5): 495-500.

25. Lewis BP, Burge CB, Bartel DP. Conserved seed pairing, often flanked by adenosines, indicates that thousands of human genes are microRNA targets. Cell. 2005; 120(1): 15-20.

26. Landgraf P, Rusu M, Sheridan R, Sewer A, Iovino N, Aravin A, et al. A mammalian microRNA expression atlas based on small RNA library sequencing. Cell. 2007; 129(7): 1401-1414.
27. Sethupathy P, Collins FS. MicroRNA target site polymorphisms and human disease. Trends Genet. 2008; 24(10): 489-497.

28. Rademakers R, Eriksen JL, Baker M, Robinson $\mathrm{T}$, Ahmed Z, Lincoln SJ, et al. Common variation in the miR-659 binding-site of GRN is a major risk factor for TDP43-positive frontotemporal dementia. Hum Mol Genet. 2008; 17(23): 36313642 .

29. Wang G, van der Walt JM, Mayhew G, Li YJ, Züchner S, Scott WK, et al. Variation in the miRNA-433 binding site of FGF20 confers risk for Parkinson disease by overexpression of alpha-synuclein. Am J Hum Genet. 2008; 82(2): 283-289.

30. Orr HT, Zoghbi HY. Trinucleotide repeat disorders. Annu Rev Neurosci. 2007; 30: 575-621.

31. Bilen J, Liu N, Burnett BG, Pittman RN, Bonini NM. MicroRNA pathways modulate polyglutamine-induced neurodegeneration. Mol Cell. 2006; 24(1): 157-163.

32. Schaefer A, O'Carroll D, Tan CL, Hillman D, Sugimori M, Llinas R, et al. Cerebellar neurodegeneration in the absence of microRNAs. J Exp Med. 2007; 204(7): 1553-1558.

33. Kim JM, Hong S, Kim GP, Choi YJ, Kim YK, Park SS, et al. Importance of low-range CAG expansion and CAA interruption in SCA2 Parkinsonism. Arch Neurol. 2007; 64(10): 15101518.

34. Karres JS, Hilgers V, Carrera I, Treisman J, Cohen SM. The conserved microRNA miR- 8 tunes atrophin levels to prevent neurodegeneration in Drosophila. Cell. 2007; 131(1): 136-145.

35. Waerner T, Gardellin P, Pfizenmaier K, Weith A, Kraut N. Human RERE is localized to nuclear promyelocytic leukemia oncogenic domains and enhances apoptosis. Cell Growth Differ. 2001; 12(4): 201-210.

36. Wells RD, Ashizawa T, Eds. Genetic Instabilities and Neurological Diseases, 2nd ed. Burlington, MA: Academic Press, 2006.

37. Sakamoto N, Ohshima K, Montermini L, Pandolfo M, Wells RD. Sticky DNA, a self-associated complex formed at long GAA*TTC repeats in intron 1 of the frataxin gene, inhibits transcription. J Biol Chem. 2001; 276(29): 27171-27177. 
38. Marsh JL, Walker H, Theisen H, Zhu YZ, Fielder T, Purcell J. et al. Expanded polyglutamine peptides alone are intrinsically cytotoxic and cause neurodegeneration in Drosophila. Hum Mol Genet. 2000; 9(1): 13-25.

39. Todd PK, Paulson HL. RNA-mediated neurodegeneration in repeat expansion disorders. Ann Neurol. 2010; 67(3): 291-300.

40. Liquori CL, Ricker K, Moseley ML, Jacobsen JF, Kress W, Naylor SL, et al. Myotonic dystrophy type 2 caused by a CCTG expansion in intron 1 of ZNF9. Science. 2001; 293(5531): 864-867.

41. Pascual M, Vicente M, Monferrer L, Artero R. The Muscleblind family of proteins: An emerging class of regulators of developmentally programmed alternative splicing. Differentiation. 2006; 74(2-3): 65-80.

42. Mankodi A, Urbinati CR, Yuan QP, Moxley RT, Sansone V, Krym M, et al. Muscleblind localizes to nuclear foci of aberrant RNA in myotonic dystrophy types 1 and 2. Hum Mol Genet. 2001; 10(19): 2165-2170.

43. Jin P, Duan R, Qurashi A, Qin Y, Tian D, Rosser TC, et al. Pur alpha binds to rCGG repeats and modulates repeat-mediated neurodegeneration in a Drosophila model of fragile X tremor/ataxia syndrome. Neuron. 2007; 55(4): 556-564.

44. Timchenko LT, Miller JW, Timchenko NA, DeVore DR, Datar KV, Lin L, et al. Identification of a (CUG)n triplet repeat RNA-binding protein and its expression in myotonic dystrophy. Nucleic Acids Res. 1996; 24(22): 4407-4414.

45. White MC, Gao R, Xu W, Mandal SM, Lim JG, Hazra TK, et al. Inactivation of hnRNP K by expanded intronic AUUCU repeat induces apoptosis via translocation of PKCdelta to mitochondria in spinocerebellar ataxia 10. PLoS Genet. 2010; 6(6): e1000984. doi: 10.1371/journal.pgen. 1000984.

46. Daughters RS, Tuttle DL, Gao W, Ikeda Y, Moseley ML, Ebner TJ, et al. RNA gain-of-function in spinocerebellar ataxia type 8. PLoS Genet. 2009; 5(8): e1000600. doi: 10.1371/journal. pgen. 1000600 .

47. Sellier C, Rau F, Liu Y, Tassone F, Hukema RK, Gattoni R, et al. Sam68 sequestration and partial loss of function are associated with splicing alterations in FXTAS patients. EMBO J. 2010; 29(7): 1248-1261.
48. Rudnicki DD, Holmes SE, Lin MW, Thornton CA, Ross CA, Margolis RL. Huntington's disease--like 2 is associated with CUG repeatcontaining RNA foci. Ann Neurol. 2007; 61(3): 272-282.

49. Batra R, Charizanis K, Swanson MS. Partners in crime: Bidirectional transcription in unstable microsatellite disease. Hum Mol Genet. 2010; 19(R1): R77-R82.

50. Zu T, Gibbens B, Doty NS, Gomes-Pereira M, Huguet A, Stone MD, et al. Non-ATG-initiated translation directed by microsatellite expansions. Proc Natl Acad Sci USA. 2011; 108(1): 260-265.

51. van Eyk CL, McLeod CJ, O'Keefe LV, Richards RI. Comparative toxicity of polyglutamine, polyalanine and polyleucine tracts in Drosophila models of expanded repeat disease. Hum Mol Genet. 2012; 21(3): 536-547.

52. Lomen-Hoerth C, Anderson T, Miller B. The overlap of amyotrophic lateral sclerosis and frontotemporal dementia. Neurology. 2002; 59(7): 1077-1079.

53. La Spada AR, Taylor JP. Repeat expansion disease: Progress and puzzles in disease pathogenesis. Nat Rev Genet. 2010; 11(4): 247-258.

54. Mackenzie IR, Bigio EH, Ince PG, Geser F, Neumann M, Cairns NJ, et al. Pathological TDP43 distinguishes sporadic amyotrophic lateral sclerosis from amyotrophic lateral sclerosis with SOD1 mutations. Ann Neurol. 2007; 61(5): 427434.

55. Mackenzie IR, Neumann M, Bigio EH, Cairns NJ, Alafuzoff I, Kril J, et al. Nomenclature for neuropathologic subtypes of frontotemporal lobar degeneration: Consensus recommendations. Acta Neuropathol. 2009; 117(1): 15-18.

56. Boxer AL, Mackenzie IR, Boeve BF, Baker M, Seeley WW, Crook R, et al. Clinical, neuroimaging and neuropathological features of a new chromosome 9p-linked FTD-ALS family. J Neurol Neurosurg Psychiatry. 2011; 82(2): 196-203.

57. van Es MA, Veldink JH, Saris CG, Blauw HM, van Vught PW, Birve A, et al. Genome-wide association study identifies 19p13.3 (UNC13A) and 9 p21.2 as susceptibility loci for sporadic amyotrophic lateral sclerosis. Nat Genet. 2009; 41(10): 1083-1087. 
58. Van Deerlin VM, Sleiman PM, Martinez-Lage M, Chen-Plotkin A, Wang LS, Graff-Radford $\mathrm{NR}$, et al. Common variants at $7 \mathrm{p} 21$ are associated with frontotemporal lobar degeneration with TDP-43 inclusions. Nat Genet. 2010; 42(3): 234-239.
59. Arocena DG, Iwahashi CK, Won N, Beilina A, Ludwig AL, Tassone F, et al. Induction of inclusion formation and disruption of lamin $\mathrm{A} / \mathrm{C}$ structure by premutation CGG-repeat RNA in human cultured neural cells. Hum Mol Genet. 2005; 14(23): 3661-3671. 
